\title{
Programa de prevención y control de la transmisión vertical del VIH en Cuba. Enero de 1986 - diciembre de 2007
}

\author{
Ida González N., Manuel Díaz J., Denis Verdasquera C. y Jorge Pérez Á.
}

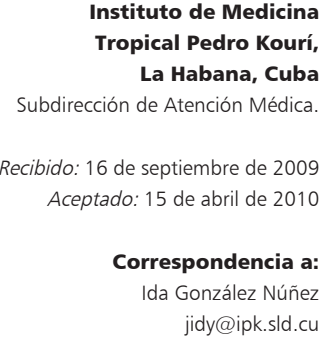

jidy@ipk.sld.cu

\author{
Prevention and control of vertical HIV transmission program in Cuba. \\ January 1986 - December 2007
}

The HIV/AIDS epidemic continues to increase in spite of the strategies developed, including the use of HAART. One of the vulnerable populations is the child of HIV+ mothers. In Cuba, a prevention and control program of HIV infection was implemented in 1986 and one of the key aims was to reduce perinatal infection. In this study, the medical records of all 314 children born to HIV/AIDS positive mothers between January 1986 to December 2007 are described. Our aim was to determine the relationship between Program compliance and prevention of vertical transmission (VT). A total of 248/314 infants completed a full virological evaluation at the time of this report $32(12.9 \%)$ were HIV-infected. In these 32 children, mortality due to AIDS related events was $31.2 \%$, of which $60 \%$ died before their first year of life. VT rate decreased from $50,0 \%$ to $14,6 \%$ after implementation of prophylactic caesarean section and to $2.2 \%$ in 99 women complying with the new adopted program including HAART antiviral therapy in pregnant woman, caesarean section, and AZT to the newborn. The effectiveness of combining a caesarean section and HAART was $95.2 \%$. The prevention program for HIV/AIDS vertical transmission implemented in Cuba has been effective in reducing VT, similar to other worldwide reports.

Key words: HIV/AIDS, vertical transmission.

Palabras clave: VIH/SIDA, transmisión vertical.

\section{Introducción}

L a forma más eficaz y económica de enfrentar en forma global el problema de la infección pediátrica por el virus de inmunodeficiencia humana (VIH) es reducir la transmisión materno infantil. El panorama ha cambiado sustancialmente en los países donde se ha podido introducir con éxito la terapia anti-retroviral (TARV) altamente activa. Los niños infectados por el VIH ahora sobreviven y llegan a la adolescencia y también a la edad adulta. En consecuencia, los retos que plantea la atención de la infección por el VIH también han evolucionado y ahora incluyen, además, a los desafíos de la atención de una enfermedad crónica.

Aún existen importantes obstáculos para ampliar la atención a la población pediátrica, por ejemplo: las limitaciones para detectar la infección por el VIH, la falta de pruebas diagnósticas sencillas para uso pediátrico, la ausencia de recursos humanos capacitados, el conocimiento insuficiente de la eficacia de la TARV en niños y su escasa promoción, la experiencia limitada con guías terapéuticas estandarizadas simplificadas, la falta de disponibilidad de anti-retrovirales en formulaciones pediátricas. En consecuencia, el número de niños que han comenzado la TARV en entornos con recursos limitados es muy bajo. La necesidad de tratar cada vez más niños infectados por el VIH resalta la importancia de poner en primer lugar la prevención de la transmisión del virus de la madre al hijo.

La transmisión vertical (TV) es la vía de adquisición de la infección por el VIH de más de $90 \%$ de los niños infectados en el mundo y en los países subdesarrollados constituye prácticamente el $100 \%$. Se estima que en el año 2007, 33,2 millones de personas entre adultos y niños padecían la infección por VIH/SIDA; 2,5 millones fueron nuevas infecciones y 2,1 millones murieron por esta causa ${ }^{1}$.

Las estadísticas mundiales sobre la morbi-mortalidad por el VIH/SIDA en niños aún son alarmantes. En el año 2007 se reportaron 2,5 millones de niños menores de 15 años que padecen la infección por VIH/SIDA, 420.000 fueron nuevas infecciones y 330.000 fallecieron según reportes de la UNICEF-VIH/SIDA del año $2007^{2}$. En 1999, en Botswana, el SIDA fue causante de $40 \%$ de todas las defunciones de niños bajo cinco años de edad. Otras cifras comparables en el mismo grupo de edad son: $35 \%$ en Zimbabwe; $25 \%$ en Namibia y Swazilandia, y más de $20 \%$ en Kenya, Sudáfrica y Zambia ${ }^{3}$. 
Así mismo estudios prospectivos han demostrado que la mediana de supervivencia es hasta los 96 meses de edad $^{5-8}$, observándose un deterioro inmunológico precoz $^{9-11}$.

La administración de TARV a la madre durante el embarazo y el parto, resolver el parto por cesárea y administrar AZT en las primeras seis semanas de vida al recién nacido han permitido reducir la tasa desde $25-30 \%$ hasta $\sim 2 \%$.

Respecto a la cesárea programada a las 38 semanas de gestación, se sabe que disminuye el riesgo de transmisión en niños nacidos de madres infectadas por el VIH que no recibieron TARV. Actualmente se discute si representa un aporte adicional en la reducción de la TV en mujeres con tratamiento combinado y carga viral (CV) indetectable. La operación cesárea es una indicación precisa en aquellas mujeres que a pesar del tratamiento ARV, no consiguen una CV suficientemente baja: menor de 1.000 copias ARN/ml sangre.

La eficacia demostrada de la profilaxis de la TV debería sensibilizar a los médicos a realizar un tamizaje de la infección VIH en mujeres en edad fértil. La identificación antes o durante el embarazo de mujeres con infección por VIH es fundamental para poder administrar un tratamiento óptimo, tanto a la madre infectada como a su descendencia, y prevenir la transmisión. La identificación de los recién nacidos expuestos permite el inicio de la profilaxis frente a las infecciones oportunistas (neumonía por $P$. jiroveci, ) y una valoración diagnóstica precoz de los lactantes infectados, que permitirá la instauración de un tratamiento precoz y agresivo.

La tasa de transmisión madre-hijo del VIH en Cuba, hasta el 31 de diciembre de 2007 fue de 10,1\% (32/314). En esta cifra están incluidos todos los niños hijos de madres seropositivas, independientemente de que ellos o la madre hayan recibido o no tratamiento profiláctico con AZT, ya que las medidas del programa de prevención y control del VIH/SIDA con su componente materno infantil (Protocolo cubano) fue implantado en el país a partir de 1986 y posteriormente se le fueron incorporando medidas con el objetivo de disminuir la TV, que se resumen a continuación ${ }^{12}$ :

- 1986: Suspensión de la lactancia materna.

- 1989: Indicación de parto por cesárea.

- 1997: AZT oral a la madre y al RN.

- 2001: TARV altamente activa a las gestantes con CD4 $<350$ céls $/ \mathrm{mm}^{3}$ y CV > 1.000 copias ARN/ml.

- 2003-2004: Realización de una segunda y tercera serología para VIH (en los tres trimestres) a todas las mujeres gestantes.

- 2004: RPC en papel de filtro antes de los 15 días de nacido.

- 2005: AZT ev intraparto.
El protocolo AIDS Clinical Trial Group-ACTG 076, fue el primer protocolo realizado para disminuir la TV y logró disminuir la tasa de transmisión vertical desde $25-30 \%$ a $8 \%{ }^{13-15}$.

A nivel mundial se han obtenido disminuciones significativas de la transmisión del VIH de madre a hijo (1-2\%), debido al uso de TARV altamente activa en dependencia de la carga viral durante el embarazo; a la suspensión de la lactancia materna, la realización de cesárea si la carga viral se encuentra por encima de 1.000 copias ARN $/ \mathrm{ml}$ en los momentos cercanos al nacimiento, la determinación de resistencia al virus y la realización de pruebas rápidas y TARV a mujeres con status desconocido al VIH, durante el inicio del trabajo de parto ${ }^{16-18}$.

Al cierre de diciembre de 2007 a nivel nacional se habían infectados por el VIH 1.804 mujeres; que corresponden a $19,3 \%$ del total de los casos diagnosticados en el país. (1.804 / 9.304). A partir del año 2000 se incrementan notablemente los nacimientos en mujeres seropositivas; de 68 niños en los primeros 15 años de la epidemia (1986-1999), a 246 nacimientos en el período 2000-2007 aumentando asimismo el número de casos infectados por el VIH de madre a hijo de 12 a 20 en igual período.

Descripción del programa. Al todo niño (a) que nace de madre con infección por VIH, se le efectúa a los 15 días de vida una RPC en papel de filtro; si ella es positiva, se confirma el diagnóstico mediante otra RPC en sangre total. De inmediato se comienza con tratamiento ARV, independientemente de su estado clínico e inmunológico, y se le determina: cuantificación de sub-poblaciones linfocitarias (CD3, CD4, CD8) y carga viral, para su clasificación según criterios del CDC de Atlanta, E.U.A., año 1994.

Si la primera RPC realizada es negativa, se repite a los 3 y 6 meses de edad, disponiéndose en cada caso de una primera RPC en papel de filtro y luego dos en sangre total. Si todas son negativas se establece la ausencia de infección vía vertical. Junto a cada RPC se determinan el AgP24 y anticuerpos mediante ELISA y western blott hasta descartar la infección en el lactante. Dos western blott negativos son requisito, junto a dos RPC negativas, para gestionar su matrícula en jardín infantil. Por orientaciones del programa, se controlan ELISA y western blott a los 9, 12, 18 y 24 meses de vida hasta comprobar la extinción de los anticuerpos maternos.

En todas las mujeres gestantes infectadas por VIH en nuestra casuística, captadas a partir del $1^{\circ}$ de enero de 1997 (fecha en que se incluyó esta medida en el programa), se les realizó profilaxis para disminuir la TV con AZT según protocolo ACTG 076 modificado (ya que no contábamos con AZT ev en esa fecha) utilizando $600 \mathrm{mg}$ de AZT desde las 14 a 38 semanas de gestación, momento en que se lleva a cabo la cesárea programada. Al RN se le administra AZT oral a partir de las seis u ocho horas de nacido: $2 \mathrm{mg} / \mathrm{kg}$ / 
dosis, cada seis horas, durante las primeras seis semanas de vida. A partir de julio del 2002 se normó también que a las mujeres gestantes en que se constataran CD4 menor de 350 células $/ \mathrm{mm}^{3}$ y CV mayor de 1.000 copias $/ \mathrm{ml} \mathrm{se} \mathrm{le}$ administrara junto con el AZT otros dos antiretrovirales como son $3 \mathrm{TC}+\mathrm{NVP}$ o d4T $+3 \mathrm{TC}+\mathrm{NVP}$ en el caso de mujeres gestantes con anemia o intolerantes al AZT.

Todos los niños cuya primera RPC en papel de filtro fuera negativa se chequearon cada tres meses observando su crecimiento y desarrollo.

Los niños que resultaron infectados por el VIH se les chequean su estado clínico, inmunológico y virológico hasta estabilizarlo desde estos puntos de vista. Estos niños reciben una dieta especial rica en proteínas, grasas y carbohidratos. Fueron clasificados según la clasificación del CDC de Atlanta de $1987^{20}$ hasta 1995, y a partir de 1996 en que el IPK adquirió el citómetro de flujo utilizando las técnicas estándar para triple marcaje y los anticuerpos monoclonales de Becton Dickinson ${ }^{21}$ se comenzó a realizarles el conteo de CD4 a las mujeres gestantes y a los niños que se reclasificaron entonces siguiendo los criterios del CDC de Atlanta de $1994{ }^{19}$ que incluye a los niños hasta 13 años de edad.

En la literatura médica se describe que al menos una cuarta parte de los niños infectados por el VIH que fallecen, lo hacen antes de cumplir el primer año de vida, y hasta $60 \%$ antes de llegar al segundo año de vida. En general, la mayoría fallece antes de los cinco años de edad ${ }^{22}$.

Desde que se aprobó el uso de TARV por la FDA en niños, se ha modificado espectacularmente el curso de la infección por el VIH, al reducir la replicación viral y prolongarles la vida ${ }^{13}$.

El presente trabajo tiene como objetivo evaluar la efectividad del programa de prevención y control de la transmisión vertical del VIH en Cuba y su evolución en el período estudiado.

\section{Material y Método}

Se realizó un estudio de corte transversal, de los 314 niños hijos de madres seropositivas para VIH entre el $1^{\circ}$ de enero de 1986 y el 31 de diciembre de 2007.

En el análisis de los factores asociados a la TV sólo se incluyeron los 248 niños que concluyeron el protocolo de evaluación de la infección adquirida por vía vertical pues 66 niños permanecían en estudio.

Los datos se recogieron de las historias clínicas de los niños y las madres atendidos en el Instituto de Medicina Tropical Pedro Kourí, así como de la base de datos del seguimiento de los hijos de mujeres seropositivas e infectados.

La información fue procesada mediante el Sofware Epi Info 6,04 b (CDC-USA) \& $\mathrm{WHO}^{23}$. Para el análisis de los datos se utilizaron tasas, frecuencias absolutas y relativas.
Para determinar la asociación entre los factores relacionados con el cumplimiento del programa de prevención y la TV del VIH, se calculó la razón de prevalencia (RP) con un $95 \%$ de confiabilidad. Se aplicó además la prueba de t student con similar confiabilidad, considerando valores de $\mathrm{p}<0,05$.

No se realizó procedimiento médico adicional alguno a los pacientes, garantizando de esta forma la confiabilidad de la información y la seguridad de los mismos, por lo que no fue necesaria la valoración del estudio por el Comité de Ética de la institución ni el consentimiento informado de los pacientes.

\section{Resultados}

Entre el $1^{\circ}$ de enero de 1986 y el 31 de diciembre de 2007 ocurrieron 314 nacimientos de 294 madres seropositivas para VIH en Cuba (16 mujeres parieron 2 veces y 4 tuvieron gemelos). De ellas, 293 eran infectadas por VIH-1 y sólo una por VIH-2.

De los 314 nacimientos, hijos de madres infectada con VIH/SIDA se observó una tendencia a incrementarse el número de nacimientos por año a partir de 1993 con mayor acentuación a partir del año 2000; el mayor número de niños nació en el año 2005, (52/314), 16,5\% del total de nacimientos en el período referido.

Al corte de este estudio habían concluido el protocolo para detectar infección por VIH de TV, 248 niños, de los cuales 216 resultaron negativos y 32 positivos.

La mayor cantidad de niños (59 mujeres y 57 varones), se ubicaron en el grupo entre 5-14 años, representando $36,9 \%$ del total. Aproximadamente $24,5 \%$ se localizan entre los primeros dos años de la vida. Se determinó asociación entre la edad mayor de 15 años y la infección por VIH (Tabla 1).

El total de 32 niños con infección por VIH representa una tasa de transmisión de 10,1\% (32/314), pero si calculamos la tasa de TV para los niños que tienen concluidos su estudio (n: 248) la tasa de transmisión es 12,9\% (32/248).

Al cierre del período se encontraban 22 niños con infección por VIH vivos, de los cuales a 20 se les administra TARV altamente activa y dos se mantenían asintomáticos, pues aún no han calificado como SIDA. El mayor número de niños infectados se encuentra en el grupo de 5-14 años (n: 17-54,5\%). No se encontró diferencia estadísticamente significativa en la distribución por sexo (Tabla 2).

La Tabla 3 detalla los hijos de madres infectadas por VIH que han fallecido por causas relacionadas con el SIDA (n: 10-32\%), distribuidos según sexo y edad en que se produce la defunción. Sesenta por ciento de ellos fallecieron antes de llegar al primer año de vida, $80 \%$ antes de arribar a los dos años (8/10) y 90\% antes de cumplir cinco años. Sólo un caso falleció después de los cinco años (8 años 10 meses) por el TARV que recibió 
pero clínicamente desarrolló el primer patrón evolutivo de esta enfermedad. No se detectaron en estos fallecidos, diferencias significativas en la distribución por grupos de edades entre ambos sexos.

Se confirmaron como no infectados por VIH 216 niños. El diagnóstico fue corroborado por los estudios realizados de RPC, WB y Ag P-24. Predominó en el sexo femenino las edades de 5 a 14 años y en el sexo masculino las edades de 2 a 4 años. Hubo dos fallecidos en etapa de lactantes -uno portador de una Tetralogía de Fallot y el otro por broncoaspiración de leche- que se corroboraron negativos por RPC (tres muestras, una en papel de filtro y dos en sangre total) (Tabla 4).

Al cierre del estudio aún se encontraban en estudio 66 niños (32 mujeres y 34 varones) para determinar infección o no por el VIH. De ellos (63/66-95,4\%) tenían bajo 2 años de edad. Se encontraban además (3/66-4,5\%) en el grupo entre 2 y 4 años pendientes de un resultado confirmatorio de WB para incluirlos en el grupo de niños no infectados, pero según establece el programa se deben cumplir los requerimientos de tener dos WB y dos RPC negativas. Ninguno de estos niños demostró signos o síntomas clínicos de infección por el VIH y no se detectaron diferencias significativas entre los sexos.

La tasa de TV se redujo considerablemente (de 50,0 a $14,6 \%$ ) a partir de la instauración del parto por cesárea como medida profiláctica dentro del programa de control. Sin embargo, cuando se incorporó además el tratamiento con AZT a partir de las 14 semanas, la reducción fue de $44,0 \%$ y de $47 \%$ cuando se realizó tratamiento con TARV altamente activa y cesárea, llegando a una TV de 2,2\% (Tabla 5).

La eficacia (relativa) de cada intervención se evaluó como el porcentaje de reducción de la tasa de TV con respecto a la observada en madres que no usaron AZT y cuyo parto se resolvió por vía vaginal. La simple ejecución de la cesárea logró una eficacia de 68,5\% (IC 95\%: 30,2$85,8)$. Conjuntamente con la cesárea y la administración de AZT, ya fuese tardío u oportuno, la eficacia alcanzó al 95,2\% IC: $95 \%(80,3-98,8)$ (Tabla 6).

Con respecto a la orfandad desde el comienzo de la epidemia en el período estudiado se constataron 10 niños huérfanos de ambos padres, 31, huérfanos de madre y 27 huérfanos de padre.

\section{Discusión}

La tasa de TV del VIH/SIDA en el período estudiado fue de $12,9 \%(32 / 248)$. Es una tasa alta pero en este resultado están incluidos los niños que nacieron incluso antes de aplicar el total de las medidas en el programa de prevención y control de la TV. Cuando se realizó el tratamiento con el cumplimiento correcto del programa (TARV altamente activa y cesárea) la TV fue de 2,2\%.
Tabla 1. Distribución por edad y sexo de los hijos de madres seropositivas para VIH/SIDA. Cuba, enero de 1986 - diciembre de 2007

\begin{tabular}{|lrrrr|}
\hline Edad & Femenino & Masculino & Total & $\%$ \\
\hline$<12$ meses & 21 & 21 & 42 & 13,4 \\
\hline $12-23$ meses & 16 & 19 & 35 & 11,1 \\
\hline $2-4$ años & 41 & 69 & 110 & 35,0 \\
\hline $5-14$ años & 59 & 57 & 116 & 36,9 \\
\hline $15-18$ años & 7 & 0 & 7 & 2,2 \\
\hline $19-21$ años & 3 & 0 & 3 & 0,9 \\
\hline 21 años & 1 & 0 & 1 & 0,3 \\
\hline Total & 148 & 166 & 314 & 100,0 \\
\hline$\chi^{2}=17,44$ & $p=0,007$. & & & \\
\hline
\end{tabular}

Tabla 2. Distribución de niños vivos con infección por VIH/SIDA según edad y sexo. Cuba, enero de 1986 - diciembre de 2007

\begin{tabular}{|lcccc}
\hline Edad & Femenino & Masculino & Total & $\%$ \\
\hline $2-4$ años & 5 & 2 & 7 & 31,8 \\
\hline $5-14$ años & 6 & 6 & 12 & 54,5 \\
\hline $15-18$ años & 1 & 0 & 1 & 4,5 \\
$19-21$ años & 1 & 0 & 1 & 4,5 \\
\hline$>21$ años & 1 & 0 & 1 & 4,5 \\
\hline Total & 14 & 8 & 22 & 100,0 \\
\hline$\chi^{2}=2,68$ & $p=0,061$. & & & \\
\hline
\end{tabular}

Tabla 3. Distribución de niños fallecidos por VIH/SIDA según edad y sexo. Cuba, enero de 1986 - diciembre de 2007

\begin{tabular}{|lcccc|}
\hline Edad & Femenino & Masculino & Total & $\%$ \\
\hline$<12$ meses & 3 & 3 & 6 & 60,0 \\
\hline 12-23 meses & 1 & 1 & 2 & 20,0 \\
\hline 2-4 años & 1 & 0 & 1 & 10,0 \\
\hline 5-14 años & 0 & 1 & 1 & 10,0 \\
\hline Total & 5 & 5 & 10 & 100,0 \\
\hline$\chi^{2}=2,00$ & $p=0,57$. & & & \\
\hline
\end{tabular}

Después de iniciar la aplicación de la TARV altamente activa en Cuba, desde julio de 2002 la supervivencia ha cambiado para los niños infectados con el VIH/SIDA. De los niños vivos al cierre del período en el estudio presentado, a $54,5 \%$ de ellos (12/22) se les ha podido prolongar la vida más allá de los cinco años de edad y $4,5 \%$ se encuentran en edades entre 19 y mayores de 21 años. Esto está asociado al seguimiento, control y tratamiento establecido por el programa ${ }^{24}$. 


\begin{tabular}{|lcccc|}
\hline \multicolumn{5}{c}{ Tabla 4. Niños negativos al VIH/SIDA hijos de madres seropositivas para } \\
VIH/SIDA. Cuba, enero de $\mathbf{1 9 8 6}-$ diciembre de 2007
\end{tabular}

\begin{tabular}{|c|c|c|c|c|c|}
\hline \multirow[t]{2}{*}{ Uso de TARV } & \multirow{2}{*}{$\begin{array}{c}\text { Tipo de } \\
\text { parto }\end{array}$} & \multirow{2}{*}{$\begin{array}{c}\text { Madres } \\
\text { VIH + }\end{array}$} & \multirow{2}{*}{$\begin{array}{l}\text { Niños } \\
\text { VIH + }\end{array}$} & \multicolumn{2}{|c|}{ Tasa de transmisión } \\
\hline & & & & $\%$ & IC $95 \%$ \\
\hline Ninguno & Vaginal & 36 & 18 & 50,0 & $32,8-7,1$ \\
\hline Ninguno & Cesárea & 41 & 6 & 14,6 & $3,34-25,9$ \\
\hline Tardío & Cesárea & 47 & 4 & 8,5 & $2,3-16,7$ \\
\hline AZT & Cesárea & 33 & 2 & 6,0 & $0,2-14,6$ \\
\hline TARV & Cesárea & 91 & 2 & 2,2 & $0,08-31$ \\
\hline Total & & 248 & 32 & 12,9 & $6,6-19,1$ \\
\hline
\end{tabular}

\begin{tabular}{|c|c|c|c|}
\hline \multicolumn{4}{|c|}{$\begin{array}{l}\text { Tabla 6. Eficacia del uso de AZT y TARGA y el } \\
\text { parto por cesárea. } \\
\text { Cuba, enero de } 1986 \text { - diciembre de } 2007\end{array}$} \\
\hline \multirow[t]{2}{*}{ Uso de TARV } & \multirow{2}{*}{$\begin{array}{l}\text { Tipo de } \\
\text { parto }\end{array}$} & \multicolumn{2}{|c|}{ Eficacia } \\
\hline & & $\%$ & IC del $\mathbf{9 5 \%}$ \\
\hline Ninguno & Cesárea & 68,5 & $30,2-85,8$ \\
\hline Tardío & Cesárea & 80,1 & $47,0-92,5$ \\
\hline AZT & Cesárea & 86,9 & $48,5-96,7$ \\
\hline TARV & Cesárea & 95,2 & $80,3-98,8$ \\
\hline
\end{tabular}

Debe realizarse el diagnóstico de VIH lo antes posible a todo RN hijo de madre que tiene la infección y comenzar tratamiento precozmente con el objetivo de evitar la replicación viral y el deterioro inmunológico ${ }^{25,26}$.

Se conoce que la cesárea sin ninguna otra medida profiláctica, disminuye la transmisión del VIH de madre a hijo en $50 \%$, si no hay rotura de membranas de más de 4 horas $^{27-30}$. Este hecho lo demostramos también en nuestra casuística.

En Cuba, el mayor porcentaje de las mujeres emba- razadas es captado en su área de salud antes de las 13,6 semanas de gestación (captación precoz) y se incluye la detección de anticuerpos contra el VIH entre los exámenes rutinarios del primero, segundo y tercer trimestre. Esto posibilita la indicación oportuna de TARV en la mujer gestante con infección por VIH. En nuestro país la cobertura del tratamiento ARV de la mujer embarazada seropositiva para VIH es de $100 \%{ }^{24}$, mientras en la mayor parte del mundo subdesarrollado los progresos en la ampliación del acceso a tratamientos de prevención ARV han sido muy lentos, estimándose que sólo $10 \%$ de las mujeres embarazadas e infectadas dispone de este servicio ${ }^{31}$. En Burkina, Fasso, Etiopia, Malawi, Nigeria y Sudáfrica menos de $1 \%$ de las mujeres infectadas por el VIH que dieron a luz en el 2003 tuvieron acceso a tratamiento para prevenir la TV. En Camboya, Myanmar y Viet Nam la cobertura es inferior a $3 \%{ }^{31}$.

Con el análisis estadístico realizado se concluyó que la no intervención, es decir parto vaginal y no uso de AZT, determinó una tasa de TV de 50,0\%. El simple hecho de evitar el nacimiento por vía vaginal logró llevar la tasa de TV a 14,6\%. Al añadirse el uso del AZT, ya sea tardío o correctamente y cesárea la tasas de TV fueron 8,5 y $6,0 \%$, respectivamente y en las mujeres gestantes con TARV altamente activa y cesárea la tasa de TV fue $2,2 \%$. No hay casos para evaluar el simple uso del AZT sin cesárea porque se necesitaría de un grupo control de mujeres seropositivas que quedarían expuestas al riesgo por permitir el paso del niño por el canal del parto, lo cual es éticamente inaceptable.

Aunque en el parto por cesárea las complicaciones son más comunes que si el parto ocurre por vía vaginal ${ }^{28,30-32}$, en nuestro medio se realiza esta modalidad de nacimiento, a las 38 semanas de la gestación, antes del inicio del trabajo de parto y hasta el momento no hemos constatado complicaciones ni muertes maternas por esta causa.

La simple ejecución de la cesárea logró un éxito de $68,5 \%$ y la eficacia de la combinación de cesárea con AZT aplicado tardíamente fue de $80,1 \%$; cesárea y AZT aplicada oportunamente alcanzó una eficacia de 86,9\%. Aplicando cesárea y TARV de alta actividad la eficacia alcanzó 95,2\%.

Analizando estos resultados se concluye que la realización de la cesárea fue el factor que mayor incidencia tuvo en la disminución de la tasa de TV. El uso de AZT o TARV altamente activa añadido a la cesárea logró eficacias crecientes.

Múltiples impedimentos obstaculizan la implantación en los países subdesarrollados de programas preventivos como el cubano. La cobertura insuficiente de los servicios de atención prenatal, la falta de recursos financieros y humanos, el escaso conocimiento del estado serológico entre las mujeres en edad de procrear, el miedo al estigma, la discriminación y la tendencia de muchas mujeres en 
los países de ingresos bajos y medianos a dar a luz en el hogar en vez de un centro de salud, son razones de tal fracaso. No obstante, constituyó un compromiso de las Naciones Unidas reducir la proporción de lactantes infectados por el VIH en 20\% para el 2005, y en 50\% para el 2010, lo cual no será posible sin un aumento inmediato y sustancial de las actividades como la ampliación de los servicios de prevención primaria del VIH para las mujeres en edad fecunda; el acceso al asesoramiento y a pruebas voluntarias para las mujeres embarazadas; la instauración de servicios integrales de salud reproductiva y el uso de profilaxis anti-retroviral para prevenir la $\mathrm{TV}^{32}$.

\section{Resumen}

La epidemia del VIH/SIDA sigue aumentando significativamente a pesar de las estrategias desarrolladas, incluyendo el desarrollo de la terapia anti-retroviral (TARV) altamente activa. Una de las poblaciones vulnerables a esta infección es los hijos de madres con infección por VIH, que no sólo están expuestos a la infección sino también a quedar huérfanos. En Cuba, desde el año 1986 se implementó un programa de prevención y control que tiene entre sus objetivos disminuir la transmisión vertical (TV) del VIH. En este estudio se analizaron los 314 niños nacidos de madres infectadas por VIH/SIDA desde el $1^{\circ} \mathrm{de}$ enero de 1986 al 31 de diciembre de 2007 en las diferentes maternidades del país, con el objetivo de determinar, mediante un estudio de corte transversal, la relación entre el cumplimiento de las medidas implantadas por el programa de prevención y la TV de la enfermedad. De los 314 niños nacidos, 248 tenían los estudios concluidos estando 32 niños infectados, 216 no infectados por el VIH y 66 niños se encontraban aún en estudio. La mortalidad por causas relacionadas al SIDA fue del $31,2 \%$, y $60 \%$ de ellos fallecieron antes de arribar al primer año de vida. La tasa de TV se redujo considerablemente (de 50,0 a 14,6\%) a partir de la instauración del parto por cesárea como medida profiláctica dentro del programa de control y 99 mujeres tuvieron $2,2 \%$ de TV cuando se cumplieron las últimas medidas del programa. La eficacia de la combinación del parto por cesárea y la administración de TARV fue de $95,2 \%$. Se concluyó que el programa de prevención y control del VIH/SIDA con su componente materno infantil y las distintas medidas que se le han ido adicionando ha sido efectivo en la reducción de la TV comportándose conforme a lo descrito internacionalmente.

\section{Referencias}

1.- AIDS Epidemia Update. Geneva: UNAIDS; 2007.

2.- González Núñez I, Díaz Jidy M, Transmisión Vertical del VIH/SIDA. En: Pérez Ávila J, Pérez Correa D, Millán Marcelo JC, Orta Gutiérrez M. Pautas cubanas para el tratamiento antirretroviral en los pacientes con VIH/SIDA. La Habana: MINSAP: PNUD: IPK; 2004. pp 47-64.

3.- American Academy of Pediatrics: Evaluation and medical treatment of the HIV-exposed infants. Pediatrics 1997; 99 (6): 909-17.

4.- Connor E M, Sperling R S, Gelber R, Kiselev P, Scott G, O'Sullivan M J, et al. Reduction of maternal infant transmission of human immunodeficiency virus type 1 with zidovudine treatment. N Engl J Med 1994; 331: 1173-80.

5.- Fiscus S A, Adimora A A, Schoenbach V J, Lim W, McKinney R, Rupar D, et al. Perinatal HIV infection and the effect of zidovudine therapy on transmission in rural and urban countries. JAMA 1996; 275: 1483-8.

6.- Vélez G L. VIH: tratamiento de la mujer embarazada y prevención de la transmisión vertical. En: Vélez A H, Rojas M W, Bornero R J, Restrepo M J, editores. Manual de VIH/ SIDA y otras infecciones de transmisión sexual. Medellín: CIB; 2005: 125-9.

7.- Health information for HIV positive pregnant women. HIV during pregnancy labour and delivery and after birth. AIDS info January 2006]. Disponible en: http: //www.aidsinfo.nih. gov. (accedido 12 marzo 2006).

8.- Achievements in Public Health: reduction in perinatal transmission of HIV infection-United States, 1986-2005. MMWR Morb Mortal Wkly Rep 2006; 55 (21): 592-8. Disponible en: http:// www cdc.gov/mmwr/preview/mmwrhtm/ $\mathrm{mm} 5221 \mathrm{a} 3 . \mathrm{htm}$. [accedido 30 junio 2006].

9.- Centers for Disease Control and Prevention.1993 revised classification system for human inmunodefiency virus infection in children less 13 years of age. MMWR Morb Mortal Wkly Rep 1994; 43 (RR-12): 12-5.

10.- Centers for Disease Control and Prevention. Revision of the CDC surveillance case definition for acquired immunodeficiency syndrome MMWR Morb Mortal Wkly Rep 1987; 36 (Suppl 15): 15- 135.

11.- Pérez L, Álvarez A, Torranzo Y, Madagan R, Perdomo Y, Vidal I, Pérez J. Evaluación de la capacidad de reconocimiento de los anticuerpos monoclonales conjugados:ior T3, T4 y T8. Rev Cub Hematol Inmunol Hemoter 2000; 16: 62-6.

12.- Dabis F, Ekpini ER. HIV-1/AIDS and maternal and child health in Africa. Lancet 2002; 359: 2097-104.

13.- Ramos Amador JT. Infección por VIH en Pediatría aspectos generales. En GonzálezGarcía J, Moreno Guillén S, Rubio García R, editores. Infección por VIH 2000. Madrid: Doyma; 2001;pp 11-46.

14.- UNICEF. La transmisión del VIH de madre a hijo: hoja de datos de la UNICEF. Ginebra: UNICEF; 2007.

15.- Walter N, Schwartlander B, Bryce J. Meeting international goals in child survival and HIV/ AIDS. Lancet 2002; 360: 284-9.

16.- Bulterys M, Fowler MG. Prevention of HIV infection in children. Pediatr Clin Nort Am 2000; 47: 241-60.

17.- Scarlatti G. Pediatric HIV infection. Lancet 1996; 348: 863-8.

18.- Barnhart HX, Caldwell MB, Thomas P, Mascola L, Ortiz I, Hsu HW, et al. Natural history of human immunodeficiency virus disease in perinatally infected children: an analysis from the Pediatric Spectrum of Disease Project. Pediatrics 1996; 97: 710-6.

19.- Tovo PA, de Martino M, Gabiano C, and the Italian Register for HIV infection in children. Prognostic factors and survival in children with human immunodeficiency virus type-1 infection. Lancet 1992, 339: 1249-53.

20.- Mc Intyre J, Gray G. What can we do to reduce mother to child transmission of HIV? Br Med J 2002; 324: 218-21.

21.- Blanche S, Newell M L, Mayaux M J, Dunn D T, Teglas J P, Rouzioux C, et al. Morbidity and mortality in European children vertically infected by HIV-1: The 
French Pediatric HIV Infection Study Group and European Collaborative Study. J Acquir Immune Defic Syndr Hum Retrovirol 1997; 14: 509-16.

22.- Resino S, Gurbindo M D, Bellón J M, Sánchez Ramión S, Muñoz Fernández M A. Predictive markers of clinical outcome in vertically HIV-1 infected infants, a prospective longitudinal study. Pediatr 2000; 47: 509-16.

23.- Dean AG, Dean JA, Coulombier D, Brendel KA, Smith DC, Burton HA, Epi Info. Version 6,04 b: a word processing, database, and statistics program for public health on IBM compatible microcomputers. CDC: Atlanta; 2000.

24.- Plan estratégico nacional ITS/VIH/SIDA, 2001-2006. Ciudad de la Habana: MINSAP; 1997.

25.- Manual Práctico de la Infección por VIH en el
Niño. 2da ed. Barcelona: Prous Science; 2000.

26.- European Collaborative Study. Mother-to-child transmission of HIV infection in the era of highly active antiretroviral therapy. Clin Infect Dis 2005; 40 (3): 458-65.

27.- Riley L E, Greene M F. The mode of delivery and the risk of vertical transmission of human immunodeficiency virus type- 1 - a meta-analysis of 15 prospective cohort studies. The International Perinatal HIV Group. N England J Med 1999; 340 (13): 977-87.

28.- Roman J, Bakos O, Cnattingius S. Pregnancy outcomes by mode of delivery among term breech births: Swedish experience 1987-1993. Obstet Gynecol 1998; 92 (6): 945-50.

29.- Gregory K D, Henry O A, Ramicone E, Chan L S, Platt L D. Maternal and infant complications in high and normal weight infants by method of delivery. Obstet Gynecol 1998; 92 (4 Pt 1): 507-13.

30.- ACOG Committee Opinion: Scheduled caesarean delivery and the prevention of vertical transmission of HIV infection. Number 234, May 2000 (replaces number 219, August 1999). Int J Gynaecol Obstet 2001; 73 (3): 279-81.

31.- Hernández E M. Prevención, diagnóstico y tratamiento de la infección por el VIH en el niño. En: Vélez A H, Rojas M W, Bornero R J, Restrepo M J, editores. Manual de VIH/ SIDA y otras infecciones de transmisión sexual. Medellín: CIB; 2005; 17-34.

32.- ONUSIDA. Informe sobre los progresos realizados en la respuesta mundial a la epidemia de VIH/SIDA, 2003. Ginebra. Disponible en http://www.unaids.org/html/pub/topics/ ungass2003/ungass_report_2003_sp_pdf.pdf 